\title{
EARLY LOADING OF SHORT AND STANDARD-LENGTH DENTAL IMPLANTS RETAINING MANDIBULAR KENNEDY CLASS I REMOVABLE PARTIAL DENTURES
}

\author{
Mohamed Ahmed Alkhodary* and Abeer Mohamed Ettesh ${ }^{* *}$
}

\begin{abstract}
Introduction: This study clinically evaluated early loaded short dental implants compared to early long dental implants assisting mandibular Kennedy Class I implant assisted removable partial denture (IARPD) with the implants placed once at the position of the missing first molar once at the position of the missing second molar.

Materials and methods: Twenty male patients, 30 to 60 years old, participated in this study and were blindly divided into 2 groups. The 10 patients of group I received one short implant placed in the mandibular right edentulous space at the site of the missing first molar, and a long implant placed in the left distal extension space at the site of the missing first molar. Group II was made of the same number of patients but with the implants placed at the sites of the missing second molars. After one year of the IARPD loading the implants were evaluated for plaque index, pocket depth, mobility, vertical bone loss, and bone density profile.
\end{abstract}

Results: No significant differences were detected in plaque index, probing depth, mobility, or vertical bone loss between the implants in the two groups, however, group I implants had more bone density profile than group II implants, especially long implants as compared to short implants.

Conclusion: The long and short implants placed at the location of the missing first molars had more bone density profile values than those placed at the place of the missing second molars, and in both cases long implants showed better bone density profile than short implants.

KEY WORDS: Short dental implants, implant assisted removable partial dentures, early loading.

\footnotetext{
* Associate Professor, Department of Prosthodontics, Faculty of Dentistry, Alexandria University, EGYPT.

** Consultant of Oral and Maxillofacial Surgery, Directorate of Outpatients Treatment Units, Alexandria University, EGYPT.
} 


\section{INTRODUCTION}

Short dental implants, $8 \mathrm{~mm}$ or less, have reached a $96 \%$ success rate, ${ }^{1}$ and were considered less invasive than standard length longer dental implants that might require adjunctive bone augmentation procedures when placed in atrophic ridges, ${ }^{2}$ in addition, short implants were thought to improve the prognosis of distal extension implant assisted removable partial dentures (IARPDS), ${ }^{3}$ especially when placed in medically compromised patients, ${ }^{4}$ and were thought to have a better prognosis when placed in the mandible than in the maxilla. ${ }^{5}$

Whether placed using single or two stage surgical protocol, short dental implants were claimed to have similar failure rates to conventional longer implants, ${ }^{6}$ however, implants of 4-7 mm length were advised to be used with caution, ${ }^{7}$ in regards to their number, location, inclination, diameter, surface treatment, and type of attachment when used to retain IARPDS in situations of poor bone quality, ${ }^{8,9}$ as they were found to have greater risk and lower predictability as compared to longer dental implants. ${ }^{10}$ Other studies recommended short implants to be used with vertical bone augmentation similar to those used with standard implants. ${ }^{11-14}$ However, in cases of atrophic mandibular partially edentulous ridges, short dental implants were found to have good therapeutic values in medium to long term clinical service, ${ }^{15}$ as they improved the RPDS support, retention, and stability. ${ }^{16-18}$

A controversy existed about the position of the implants under distal extension bases, whether to be placed mesially near the principal abutments or more distally towards the end of the saddles, and whether these bases should be used unilaterally, on separate bases, or connected by major connectors. ${ }^{19-21}$ Another controversial factor was the type of attachment of the implants to the IARPDS, where the ball abutments were found to transmit the least amount of stress to the implants and abutment teeth compared to locator and magnet attachments, when placed parallel to the most distal abutment, however, with reduced stability due to its greater freedom of movement, yet with more benefits and preservation of supporting teeth and implants when combined with mesially placed abutments..$^{22-37}$

Immediate loading of short implants placed in distal extension edentulous spaces was considered an option in the treatment protocols, once primary stability could be achieved in selected cases, ${ }^{38-41}$ also, no significant differences between the conventional loading and immediate loading of short implants were found, ${ }^{42-45}$ with either locator or ball abutment attachments in follow-up periods extending from 2 to 12 years, ${ }^{46-49}$ with preference of prognosis for implants placed in atrophic mandibles compared to atrophic maxillae. ${ }^{50-56}$

Based on the previously presented data, this study aimed at clinical evaluation of early loaded short dental implants, compared to early loaded conventional long dental implants, supporting mandibular Kennedy Class I IARPDS placed once at the position of the missing first molar once at the position of the missing second molar in the distal extension areas.

\section{MATERIALS AND METHODS}

\section{Patient selection}

Twenty male patients, 30 to 60 years old, participated in this study after understanding the procedure and having them sign an informed consent. The patient inclusion criteria included: 1) having a Class I Kennedy mandibular arch, 2) Alveolar bone not less than $10 \mathrm{~mm}$ height above the inferior alveolar canal, and a minimum ridge width of $6 \mathrm{~mm}, 3$ ) having a fully dentate maxillary arch, or an arch that could be reconstructed with fixed restorations, 4) nonsmoker, 5) non-diabetic. The patient's exclusion criteria were as follows: 1) smoker, 2) diabetic, 3) chronic cardiovascular disease, 4) bad oral hygiene 5) non responsive 
to motivation or appreciation of the treatment provided.

The participating patients were blindly divided into 2 groups:

Group I: made up of 10 patients, each received one short one implant (6 mm long and $4 \mathrm{~mm}$ width, Astra tech, Dentsply, Sirona, Germany) placed in the right edentulous space at the approximate site of the missing first molar, and a conventional long implant (10 $\mathrm{mm}$ long and $4 \mathrm{~mm}$ width, Astra tech, Dentsply, Sirona, Germany) placed in the mandibular left distal extension edentulous space also at the approximate site of the missing first molar.

Group II: made up of 10 patients, each received one short one implant (6 mm long and $4 \mathrm{~mm}$ width, Astra tech, Dentsply, Sirona, Germany) placed in the right edentulous space at the approximate site of the missing second molar, and conventional long implant (10 $\mathrm{mm}$ long and $4 \mathrm{~mm}$ width, Astra tech, Dentsply, Sirona, Germany) placed in the mandibular left distal extension edentulous space also at the approximate site of the missing second molar.

\section{Treatment planning}

Each of the participating patients had a custommade treatment plan aimed at ending up with a mandibular arch ready to receive a Kennedy Class I RPD and a reconstructed maxillary occlusal plane up to the upper second molars. Pre-operative clinical and radiographic examinations were conducted, panoramic and peri-apical $\mathrm{x}$-rays were taken for each patient, preliminary impressions of the upper and lower arches were made and mounted on an articulator for further examination of the available inter-arch space, and the orientation of the occlusal plane. The treatment sequence included: periodontal therapy, oral surgery and placement of implant fixtures, restoration of carious teeth, restoration of missing teeth with fixed partial dentures, and surveyed crowns if the restored teeth were in the path of insertion of the RPDS, and finally the patients were provided with a temporary acrylic resin RPD to be used during the healing time after implants placement.

\section{Surgical protocol}

A cone beam computed tomography (CBCT) $\mathrm{X}$-ray was made for each patient, the CBCT axial cuts were used to identify the location of the inferior alveolar nerve canal, and to construct a surgical guide with a metal sleeve at the position of implant placement. The patients were premedicated with analgesics/anti-inflammatory, Ketoprufen $150 \mathrm{mg}$, and antibiotics, Amoxicillin Clavulonic acid 625 $\mathrm{mg}$, the patients were asked to use chlorohexidine mouth wash prior to the procedures. Inferior alveolar nerve block anesthetic, HCL 2\% and adrenaline as Levonordefrin 1: 20000, was administered, a crestal incision was made on the edentulous ridge, then a full muco-periosteal flap was reflected. The surgical template was secured in place for drilling the osteotomies. Both long and short implant osteotomies were prepared using a maximum of 1100 rpm high torque motor (Osseoset 200, Nobel Biocare) irrigated with sterile water, and a drill sequence of 2-, 3.2-, and 3.7-mm drills to a depth of 10 and $6 \mathrm{~mm}$ respectively. The implants were then placed to an equicrestal position. After placement of both types of implants, as seen in figures 1 and 2 , cover screws were secured in place and left uncovered by the gingiva, then the temporary RPD was delivered and relieved over the implant sites, and patients were asked again to use mouth was, and were given post operative care instructions and medications.

\section{Prosthetic protocol and implant early loading}

After one week of implants placement, patients were recalled for suture removal and preliminary impressions making, in the following visits final impressions, registration of jaw relationships, try-in of metal frameworks and wax dentures were made 
using conventional methods. ${ }^{20,22,25,27,30,34}$ After one month of healing for both long and short implants, the ball abutments were attached to the implants, and after final impressions making, replicas of the ball abutments were secured in the impressions to be part of the master casts over which definitive prostheses were flasked including the metal housing of the ball abutments in the IARPDS metal framework as seen in figures 3-5. The IARPDS had a lingual plate major connector, and combination clasps to relieve the abutments during occlusal loading.

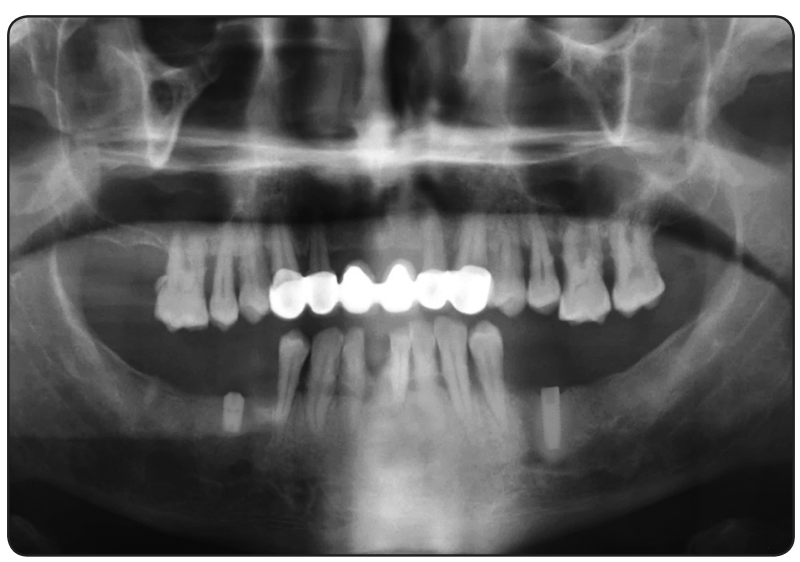

Fig. (1): Immediate post-operative panoramic x-ray of group I, short and long implants placed at the approximate location of the first molar.

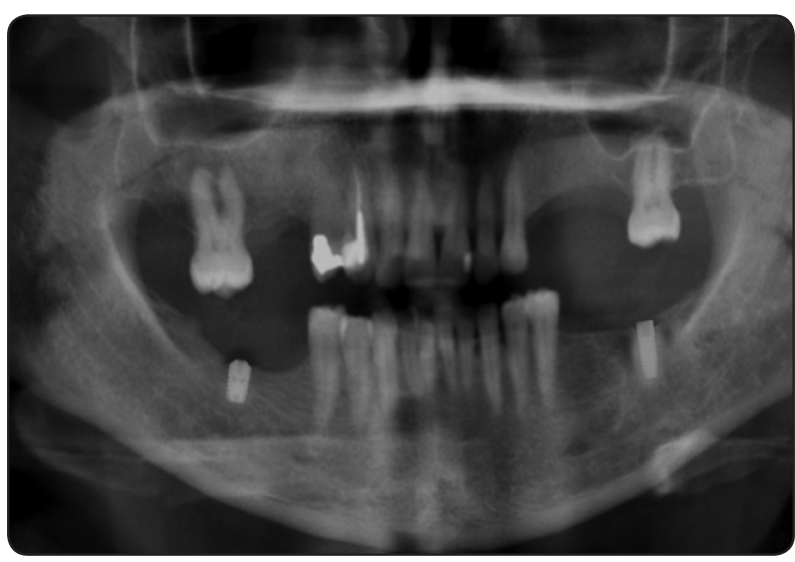

Fig. (2): Immediate post-operative panoramic x-ray of group II, short and long implants placed at the approximate location of the second molar.

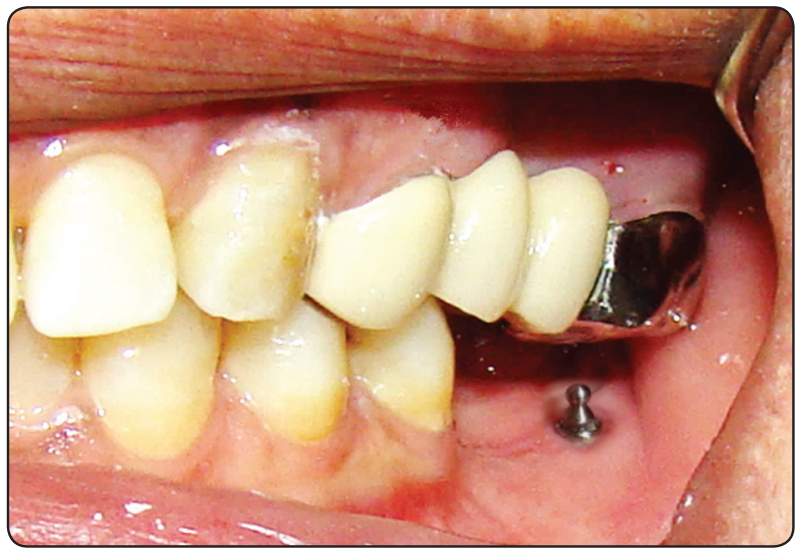

Fig. (3): Group I short implant ball abutment.

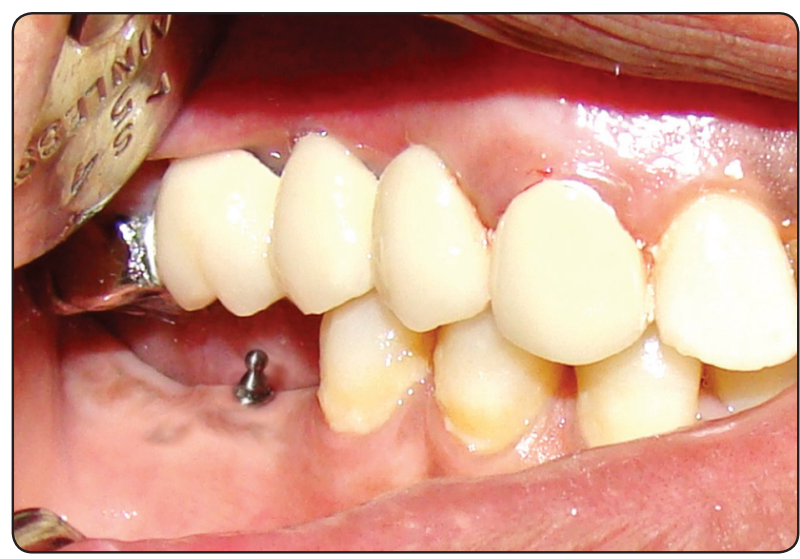

Fig. (4): Group II long implant ball abutment.

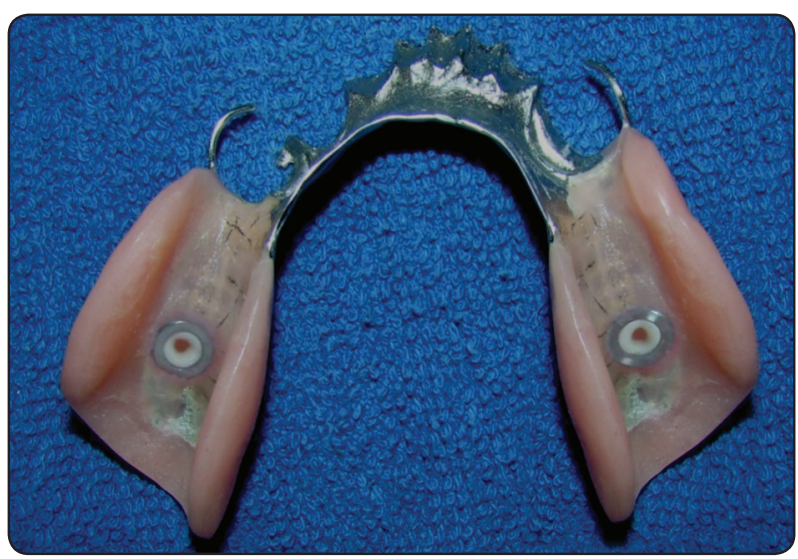

Fig. (5): One of group II definitive prostheses with metal housings and O-rings for the ball abutment in the IARPD intaglio surface. 


\section{Patient follow up}

The clinical evaluation included study of the plaque index, pocket or probing depth, implant stability using the periotest, and radiographic examination which included determination of the peri-implant vertical bone loss and bone density profile using standardized digital peri-apical x-rays and the ImageJ software. Base line measurements of implant stability and peri-implant bone vertical height and density were made, then patients were followed up after 12 months of prostheses delivery.

For assessment of the vertical bone loss, the $\mathrm{x}$-ray images were opened with the ImageJ software and the following steps were taken:

1. The scale was determined in reference to the known implant length using the set scale command in the software to convert the pixel dimensions to millimeters.

2. The distance from the shoulder of the implant (implant-abutment interface level) to the first visible bone-to-implant contact was determined by linear measurements. In addition, the length of the implant was measured in order to determine the magnification factor in the radiograph as seen in figure 6 . The measurements of the bone levels were then adjusted according to the magnification.

3. A line was drawn from the reference point in implant abutment and the first point of bone implant contact, the measurements in mm were noted both mesially and distally and the mean was calculated.

And for assessment of the bone density profile around the implants, the region of interest (ROI), as seen in figure 7 , was selected which was in close level to bone implant interface in the mesial (point 1), distal (point 2) and apical (point 3) aspects, and the degree of blackening and whitening (radiolucency and radiopacity) was expressed in numbers from 0 to 255 , and the following steps were taken:
1. The rectangle marquee tool was used to make selection of the area including the bone implant interface.

2. Form the "analyze" command in the title bar select "measure" to give mean gray value (mean density)

3. Mean Gray Value (average gray value within the selection) was the sum of the gray values of all the pixels in the selection divided by the number of pixels, then the results were saved

The abutment teeth were at the beginning of the treatment plan and their pocket depth and mobility were registered in the periodontal charts, then after one year of loading the teeth were examined again.

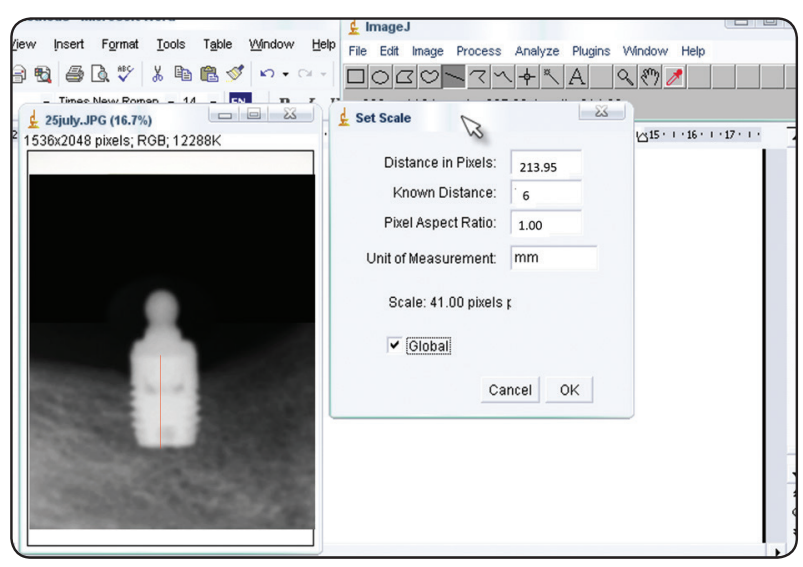

Fig. (6): The use of ImageJ software to determine the periimplant vertical bone loss

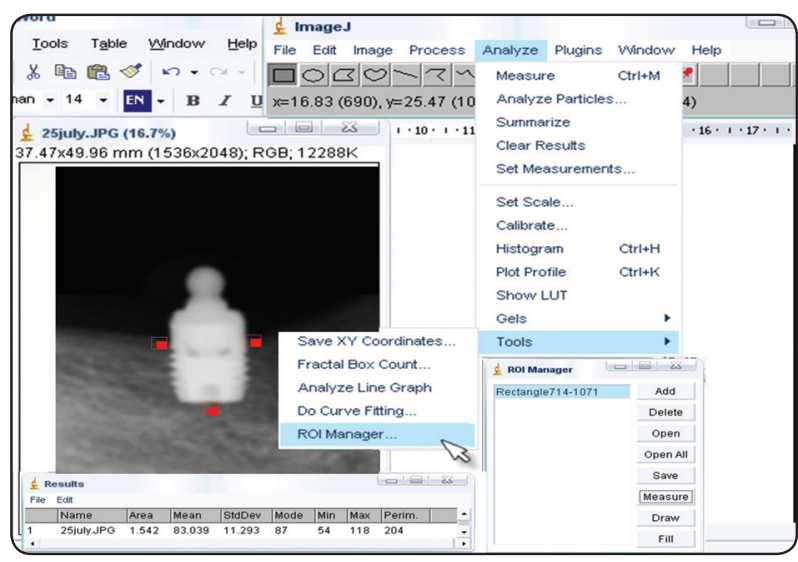

Fig. (7): The use of Image $\mathrm{J}$ software to determine the periimplant bone density profile at three points (red squares), namely: mesial, distal, and apical. 
The collected data were tabulated and statistically analyzed using the Paired T test (SPSS version 20 for windows).

\section{RESULTS}

\section{1) Plaque index:}

In both group I and II, the mean plaque index of short implants was higher than that for long implants, however, these results were statistically insignificant. (Table 1) (Fig. 8)

\section{2) Probing depth:}

For group I, the mean probing depth for long implants was greater than that for short implants, however, this result was statistically insignificant. (Table 1) (Fig. 8)

For group II, the mean probing depth for short implants was greater than that for long implants, however, this result was statistically insignificant. (Table 1) (Fig. 8)

\section{3) Mobility:}

For both group I and II the mean of periotest readings of short implants was greater than that for long implants, however, these results were statistically insignificant. (Table 1)

\section{4) Radiographic evaluation:}

\section{a) Vertical bone loss ( $\mathrm{mm})$ :}

For both group I and II the mean vertical bone loss of short implants was greater than that for long implants, however, these results were statistically insignificant. (Table 1) (Fig. 8)

\section{b) Bone density measurement:}

Comparisons within each group revealed that for group I long and short implants the mean bone density profile values at points 1,2 , and 3 after 12 months of loading were significantly greater than its values on the time of loading at the same points respectively, however, these values were significantly greater around long than short implants as seen in tables 2 and 3. The same results were obtained for group II long and short implants, where each category showed increase in its bone density profile at each of the 3 studied points, yet the long implants exhibited significant increase in its recorded bone density profiler values as also seen in tables 2 and 3 .

Comparison between groups revealed that long and short implants of group I had significantly greater bone density profile at the same studied points in separate and in overall basis as seen in table 4.

Finally, none of the abutment teeth shoed any increase in pocket depth or increased mobility.

TABLE (1): Descriptive (mean and standard deviation SD) and statistical analysis (p value) of the one year follow up of the short and long implants

\begin{tabular}{|c|c|c|c|c|c|c|c|}
\hline & & \multicolumn{3}{|c|}{ Group I } & \multicolumn{3}{|c|}{ Group II } \\
\hline & & Long implants & Short implants & $\mathrm{p}$ & Long implants & Short implants & $\mathrm{p}$ \\
\hline \multirow{2}{*}{ Plaque index } & Mean & 1 & 1.833 & \multirow{2}{*}{0.06} & 0.813 & 1.167 & \multirow{2}{*}{0.16} \\
\hline & SD & 0.204 & 0.753 & & 0.239 & 0.408 & \\
\hline \multirow{2}{*}{ Probing depth } & Mean & 1.375 & 1.333 & \multirow{2}{*}{0.88} & 1.5 & 1.583 & \multirow{2}{*}{0.75} \\
\hline & $\mathrm{SD}$ & 0.479 & 0.408 & & 0.408 & 0.376 & \\
\hline \multirow{2}{*}{ Mobility } & Mean & -6.5 & -5.3 & \multirow{2}{*}{0.07} & -6.09 & -5.4 & \multirow{2}{*}{0.06} \\
\hline & SD & 1.23 & 1.16 & & 1.22 & 1.12 & \\
\hline Vertical bone & Mean & 0.625 & 1 & \multirow{2}{*}{0.08} & 0.375 & 0.75 & \multirow{2}{*}{0.15} \\
\hline loss & SD & 0.250 & 0.316 & & 0.479 & 0.274 & \\
\hline
\end{tabular}




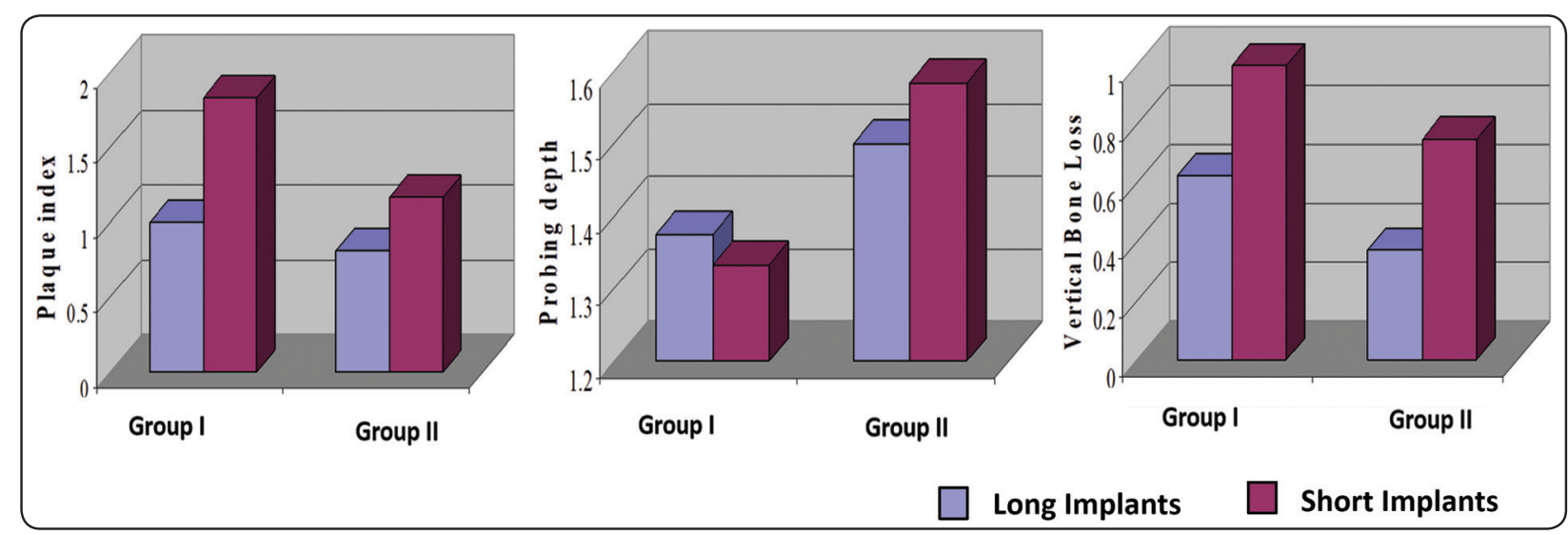

Fig. (8): bar charts of group I and II long and short implants plaque index, probing depth, and vertical bone loss.

TABLE (2): Bone density measurements pre-operatively and at 12 months.

\begin{tabular}{ccccccccc}
\hline \multirow{2}{*}{ group } & \multirow{2}{*}{ Implant } & $\begin{array}{c}\text { Mean and standard } \\
\text { deviation (SD) }\end{array}$ & \multicolumn{3}{c}{ At loading } & \multicolumn{3}{c}{12 months after loading } \\
\cline { 5 - 8 } & & Point 1 & Point 2 & Point 3 & Point 1 & Point 2 & Point 3 \\
\hline \multirow{3}{*}{ Group I } & $\begin{array}{c}\text { Long } \\
\text { implants }\end{array}$ & Mean & 65 & 48.4 & 132.4 & 152.8 & 149.8 & 219.8 \\
& & SD & 12.83 & 12.99 & 29.18 & 12.68 & 24.97 & 9.36 \\
& $\begin{array}{c}\text { Short } \\
\text { implants }\end{array}$ & Mean & 61 & 52 & 145 & 109 & 109.2 & 183.2 \\
& SD & 11.95 & 13.24 & 32.13 & 16.31 & 26.86 & 9.6 \\
\hline \multirow{3}{*}{ Group II } & implants & Mean & 57 & 56.8 & 148.4 & 95 & 95.2 & 196.4 \\
& Short & SD & 6 & 5.8 & 15.99 & 5.15 & 3.11 & 11.99 \\
& implants & Mean & 64 & 51 & 138 & 77.8 & 79.6 & 170.6 \\
\hline
\end{tabular}

TABLE (3): Statistical analysis ( $p$ value) of bone density profile within each group after one year of loading versus base line measurements at point 1,2 and 3

\begin{tabular}{ccccc}
\hline Group & Implant type & Point 1 & Point 2 & Point 3 \\
\hline \multirow{3}{*}{ Group 1 } & Long implants & 0.00 & 0.01 & 0.00 \\
& Short implants & 0.04 & 0.01 & 0.04 \\
& Long versus short implants & 0.00 & 0.01 & 0.02 \\
\hline \multirow{3}{*}{ Group II } & Long implants & 0.02 & 0.03 & 0.03 \\
& Short implants & 0.01 & 0.00 & 0.04 \\
& Long versus short implants & 0.03 & 0.04 & 0.04 \\
\hline
\end{tabular}


TABLE (4): Comparison of the bone density profile of all implants of group I versus those of group II after 1 year of loading

\begin{tabular}{|c|c|c|c|c|c|}
\hline Group & Descriptive analysis & Point 1 & Point 2 & Point 3 & Overall \\
\hline \multirow{2}{*}{ Group I } & Mean & 130.9 & 129.5 & 201.5 & \multirow{5}{*}{0.00} \\
\hline & SD & 14.5 & 25.9 & 9.48 & \\
\hline \multirow{2}{*}{ Group II } & Mean & 86.4 & 87.4 & 183.5 & \\
\hline & SD & 4.98 & 4.73 & 14.79 & \\
\hline Statistical analysis & $\mathrm{p}$ & 0.00 & 0.01 & 0.00 & \\
\hline
\end{tabular}

$P$ is significant at $\geq 0.05$

\section{DISCUSSION}

Xie et $\mathrm{al}^{3}$ stated that growth of geriatric population increased the number of partially edentulous patients and their demands of removable partial dentures (RPDS). Da Silva et $\mathrm{al}^{17}$ and Zancopé et $\mathrm{al}^{24}$ found that dental implants assisting RPDS improved their support, retention, and stability, provided better masticatory performance, and preserved the abutment teeth and their periodontium. Jagadeesh et $\mathrm{al}^{4}$ reported that short implants provided the same service as long implants under RDPDS in patients with poor bone quantity, and Telleman et $\mathrm{al}^{5}$ reported that short implants had more success in the mandibles than in maxillae of partially edentulous patients, without any bone augmentation procedures, irrespective of the implant length or surface topography but excluding from these results smoker patients. The current study reported success of short dental implants in assisting IARPDS, with plaque index, probing depth and absence of mobility similar to the long implants used in this study in a split mouth approach. The short implants used in this work had the same diameter, macro-design, and surface treatment as the long implants.

Similar to the results of this study, Sun et $\mathrm{al}^{6}$ and Lemos et $\mathrm{al}^{7}$ reported that short implants showed marginal bone loss similar to standard long implants, Torres-Alemany et $\mathrm{al}^{13}$ Further stated that the parameters of implant length, diameter, or crown/ implant ratio have not been established as being statistically significant in terms of its influence on bone or implant loss. However, this finding came in contrast to Papaspyridakos et $\mathrm{al}^{10}$ who claimed that short implants had higher rates of failure in 1 to 5 years of service as compared to conventional long implants, especially when the short implants length was less than $6 \mathrm{~mm}$, or more generally less than 10 $\mathrm{mm}$ as indicated by Abdel-Halim et al. ${ }^{14}$

In contrast to Alam-Eldein et al ${ }^{20}$ who suggested unilateral RPDS to restore class II Kennedy arches, this study used conventional design RPDS having major connectors to counteract the effects of unilateral loadings through cross arch stabilization as advocated by the finite element study of Messias et $\mathrm{al}^{21}$, and further confirmed by Shahmiri et al ${ }^{19,36}$ who found that unilateral occlusal loading generated vertical and lateral displacements of the IARPD, and resulted in destructive stresses to the prostheses and supporting abutments. Shahmiri et $\mathrm{al}^{19}$ also found that bilateral loading minimized the lateral distorting forces, but placed more strains in the occlusal rests of the principal abutments as the load moved more mesial, this finding verified the results of this study in regards to the placement of the implants in the place of the missing first molar rather than the second molar, as the vertically applied bilateral loading was along the long axes of the principal abutments, and the implants that were placed parallel to them, and being within the limits of the physiologically tolerated loads, the bone density profile of the mesially placed implants was more than the distally placed ones. Hegazy et al, ${ }^{21}$ in contrast to the 
findings of this study, suggested distal rather than placement of the implants, however, in His study, the mesially placed implants were in the premolar region, where as the mesially placed implants in this study were in the first molar region that was surrounded by thick cortical plates that dissipated the vertically applied loads, in contrast to the distally placed implants that suffered longer effort arms and more lateral twisting loading exaggerated by the upward curvature of the distal extension saddles as they approach the retromolar pads as confirmed by ELsyad et al, ${ }^{30}$ and Alkhodary ${ }^{34}$ who found that distal placement of implants, beneath distal extension partial overdentures, recorded significantly higher stresses than with their mesial placement.

Khaki and Shishehian ${ }^{26}$ found that ball abutments, similar to those used in this study, resulted in lower stability of the IARPD due to its greater freedom of movement, however, Omar et $\mathrm{al}^{22}$ and ELsyad et $\mathrm{al}^{32}$ reported that ball attachment used to retain IARPD recorded the lowest strain around abutment teeth compared to locator and magnetic attachment. Liu et $\mathrm{al}^{46}$ further added that both locator and ball attachments helped minimize the marginal bone loss around immediately loaded un-splinted implants retaining mandibular overdentures.

Immediate loading of short implants was reported in several studies of Alvira-González et $\mathrm{al}^{38}$, Weerapong $\mathrm{K}$ et $\mathrm{al}^{42}$, and Hadilou et $\mathrm{al}^{45}$ However, this study adopted a more careful approach, the early loading, based on previous studies on the mandibular numerical models,${ }^{37}$ and bone density in the posterior mandible, ${ }^{43,49,57}$ where the achieved primary stability of the short dental implants, together of one months of healing, the vertical placement of the implant, and the resilient attachment, enabled the short implants used in this study to demonstrate vertical bone loss values and surrounding bone density profile similar to the standard conventional long implants.

In conclusion, the overall one year follow up results of this study were in agreement with several other studies, where in a retrospective study of 124 cases, Grant et $\mathrm{al}^{47}$ found that short implants were able to provide an effective alternative to long implants in cases of atrophic posterior mandibles and helped to avoid bone grafting and mandibular nerve re-positioning. The same results were confirmed by El Mekawy et $a 1,{ }^{48}$ and Faot et $\mathrm{al}^{51}$ who used short implants in a split mouth approach similar to that used in this study, however, his implants were splinted, and Guida et $\mathrm{al}^{52}$ who used short implants in completely edentulous patients. The previously mentioned follow up findings were further confirmed by the results of Banihashemrad et $\mathrm{al}^{54}$ study on the stability of short implants compared to long implants using also the periotest, where the short implants showed a similar stability, and provided a successful alternative to long implants, as well as improved the patient psychology and prostheses performance as proved by Bellia et $\mathrm{al}^{55}$ and Pardo-Zamora et al. ${ }^{56}$

Finally, the limitations of the current work included some parameters that were recommended to be investigated in future research such as: involvement of larger number of patients and following them up for longer periods of clinical service, using resilient attachments of different forms and heights of to retain the IARPD, and investigating the effect of the available inter-arch space on the choice of these attachments together with the mechanical behavior of the overlying prostheses.

\section{CONCLUSION}

Taking the limitations of the current work into account, the following conclusions were listed:

1- After one year of clinical service, the early loaded short implants placed at the locations of the missing first and second molars successfully retained the IARPD similar to the standardlength long implants without failure.

2- The long and short implants placed at the location of the missing first molars had more bone density profile values than those placed at the place of the missing second molars, and in both cases long implants showed better bone density profile than short implants. 


\section{REFERENCES}

1. Kiattavorncharoen S, Boonsiriseth K, Min K, Suriyan N, Wongsirichat N. Why short implant?. Journal of Medicine and Medical Sciences. 2014 Apr;5(4):97-101.

2. Shammas M, Shakeel SK. Short Dental Implants: Its Rationale for Use. International Journal of Oral Implantology and Clinical Research. 2015.

3. Xie Q, Ding T, Yang G. Rehabilitation of oral function with removable dentures-still an option?. Journal of Oral Rehabilitation. 2015 Mar;42(3):234-42.

4. Jagadeesh KN, Verma AK, Parihar AS, Kochhar AS, Das AC, Razi MA. Assessment of the Survival Rate of Short Dental Implants in Medically Compromised Patients. The Journal of Contemporary Dental Practice. 2020 Aug $1 ; 21(8): 881$.

5. Telleman G, Raghoebar GM, Vissink A, Den Hartog L, Huddleston Slater JJ, Meijer HJ. A systematic review of the prognosis of short $(<10 \mathrm{~mm})$ dental implants placed in the partially edentulous patient. Journal of clinical periodontology. $2011 \mathrm{Jul}$;38(7):667-76.

6. Sun HL, Wu YR, Huang C, Shi B. Failure rates of short ( $\leq 10 \mathrm{~mm}$ ) dental implants and factors influencing their failure: a systematic review. International Journal of Oral \& Maxillofacial Implants. 2011 Aug 1;26(4).

7. Lemos CA, Ferro-Alves ML, Okamoto R, Mendonça MR, Pellizzer EP. Short dental implants versus standard dental implants placed in the posterior jaws: A systematic review and meta-analysis. Journal of dentistry. 2016 Apr 1;47:817.

8. Shue L, Miron RJ, Yufeng Z. Review of implant support for the distal extension removable partial dentures. JSM Dent Surg. 2016;1(1):1007.

9. Jain N, Gulati M, Garg M, Pathak C. Short implants: new horizon in implant dentistry. Journal of clinical and diagnostic research: JCDR. 2016 Sep;10(9):ZE14.

10. Papaspyridakos P, De Souza A, Vazouras K, Gholami H, Pagni S, Weber HP. Survival rates of short dental implants ( $\leq 6 \mathrm{~mm}$ ) compared with implants longer than $6 \mathrm{~mm}$ in posterior jaw areas: A meta-analysis. Clinical oral implants research. 2018 Oct;29:8-20.

11. Thoma DS, Cha JK, Jung UW. Treatment concepts for the posterior maxilla and mandible: short implants versus long implants in augmented bone. Journal of Periodontal \& Implant Science. 2017 Feb 1;47(1):2-12.
12. Assaf F, Siqueira-Ibelli G, Margonar R, Santos PL, SouzaFaloni AP, Pereira-Queiroz T. Survival of short dental implants in atrophied jaw: a systematic review. International journal of interdisciplinary dentistry. 2020 Apr;13(1):44-6.

13. Torres-Alemany A, Fernández-Estevan L, AgustínPanadero R, Labaig-Rueda C, Mañes-Ferrer JF. Clinical behavior of short dental implants: Systematic review and meta-analysis. Journal of Clinical Medicine. 2020 Oct;9(10):3271.

14. Abdel-Halim M, Issa D, Chrcanovic BR. The Impact of Dental Implant Length on Failure Rates: A Systematic Review and Meta-Analysis. Materials. 2021 Jan;14(14):3972.

15. Carosi P, Lorenzi C, Laureti M, Ferrigno N, Arcuri C. Short Dental Implants $(\leq 6 \mathrm{~mm})$ to Rehabilitate Severe Mandibular Atrophy: A Systematic Review. International Journal of Oral \& Maxillofacial Implants. 2021 Jan 1;36(1).

16. Mijiritsky E. Implants in conjunction with removable partial dentures: a literature review. Implant dentistry. 2007 Jun 1;16(2):146-54.

17. Da Silva B, Xediek Consani RL, Lopes De Oliveira GJ. Association between implants and removable partial dentures: review of the literature. Revista Sul-Brasileira de Odontologia. 2011 Jan;8(1):88-92.

18. Chatzivasileiou K, Kotsiomiti E, Emmanouil I. Implantassisted removable partial dentures as an alternative treatment for partial edentulism: a review of the literature. General dentistry. 2015 Mar 1;63(2):21-5.

19. Shahmiri R, Aarts JM, Bennani V, Das R, Swain MV. Strain distribution in a Kennedy class I implant assisted removable partial denture under various loading conditions. International journal of dentistry. 2013 Jan 1;2013.

20. Alam-Eldein AM, Abd El Fattah FE, Shakal EA. Comparative study of two different designs of partial over denture supported with distal implant for the treatment of mandibular Kennedy class II cases. Tanta Dental Journal. 2013 Aug 1;10(2):39-47.

21. Hegazy SA, Elshahawi IM, ElMotayam H. Stresses induced by mesially and distally placed implants to retain a mandibular distal-extension removable partial overdenture: a comparative study. International Journal of Oral \& Maxillofacial Implants. 2013 Apr 1;28(2).

22. Omar A, Omran M, Fouad M, Elsyad M. Effect of different attachments designs used for implant assisted mandibular distal extension RPD. An in vitro study of stresses transmitted to abutment teeth. Mansoura Journal of Dentistry. 2014;1(3):124-30. 
23. Hirata K, Takahashi T, Tomita A, Gonda T, Maeda Y. The Influence of Loading Variables on Implant Strain When Supporting Distal-Extension Removable Prostheses: An In Vitro Study. International Journal of Prosthodontics. 2015 Sep $1 ; 28(5)$.

24. Zancopé K, Abrão GM, Karam FK, Neves FD. Placement of a distal implant to convert a mandibular removable Kennedy class I to an implant-supported partial removable Class III dental prosthesis: A systematic review. The Journal of prosthetic dentistry. 2015 Jun 1;113(6):528-33.

25. Jensen C, Raghoebar GM, Kerdijk W, Meijer HJ, Cune MS. Implant-supported mandibular removable partial dentures; patient-based outcome measures in relation to implant position. Journal of dentistry. 2016 Dec 1;55:92-8.

26. Khaki MN, Shishehian A. Stress distribution in natural tooth and implant supported removable partial denture with different attachment types: A photoelastic analysis. Journal of Islamic Dental Association of IRAN (JIDAI). 2016;28(1):1.

27. Ramchandran A, Agrawal KK, Chand P. Implant-assisted removable partial denture: An approach to switch Kennedy Class I to Kennedy Class III. The Journal of the Indian Prosthodontic Society. 2016 Oct;16(4):408.

28. Bassetti RG, Bassetti MA, Kuttenberger J. Implant-Assisted Removable Partial Denture Prostheses: A Critical Review of Selected Literature. The International journal of prosthodontics. 2018 May 1;31(3):287-302.

29. Githanjali Manchikalapudi D, Prasad A, Rao L. Attitude of partial denture wearers towards implant treatment and its association with oral hygiene status, age, gender and extent of edentulousness-A cross sectional study.

30. ELsyad MA, El Ghany Kabil AA, El Mekawy N. Effect of Implant Position and Edentulous Span Length on Stresses Around Implants Assisting Claspless Distal Extension Partial Overdentures: An In Vitro Study. Journal of Oral Implantology. 2017 Apr;43(2):100-6.

31. Hirata K, Takahashi T, Tomita A, Gonda T, Maeda Y, Hirata K, Takahashi T, Tomita A, Gonda T, Maeda Y. Influence of Abutment Angle on Implant Strain When Supporting a Distal Extension Removable Partial Dental Prosthesis: An In Vitro Study. International Journal of Prosthodontics. 2017 Jan 1;30(1).

32. ELsyad MA, Omran AO, Fouad MM. Strains Around Abutment Teeth with Different Attachments Used for ImplantAssisted Distal Extension Partial Overdentures: An In Vitro Study. Journal of prosthodontics. 2017 Jan;26(1):42-7.
33. Liu Y, Zhang YN, Sasaki K, Chen XD. Influence of location of osseointegrated implant on stress distribution in implant supported longitudinal removable partial dentures: 3-dimensional finite element analysis. Int J Clin Exp Med. 2019 Jan 1;12(8):10399-410.

34. Alkhodary MA. Class II Kennedy implant assisted mandibular removable partial dentures with and without cross arch stabilization: A strain gauge in vitro study. Egyptian Dental Journal. 2020 Apr 1;66(2-April (Fixed Prosthodontics, Dental Materials, Conservative Dentistry \& Endodontics)):1173-82.

35. Messias A, Neto MA, Amaro AM, Lopes VM, Nicolau P. Mechanical Evaluation of Implant-Assisted Removable Partial Dentures in Kennedy Class I Patients: Finite Element Design Considerations. Applied Sciences. 2021 Jan;11(2):659.

36. Shahmiri R. The effect of loading conditions on a Kennedy Class I implant-assisted removable partial denture (Doctoral dissertation, University of Otago). 2011.

37. R. Moustafa, Tamer M. Nassef , M. Alkhodary, Mona K. Marei, Magdy A. Awadalla: A New Interactive 3-D Numerical Model of the Human Mandible for Peri-Implant Analysis in-Vivo Compared With Cone Beam Computed Tomography 3-D Quality. American Journal of Biomedical Engineering 2011; 1(1): 1-8

38. Alvira-González J, Díaz-Campos E, Sánchez-Garcés MA, Gay-Escoda C. Survival of immediately versus delayed loaded short implants: A prospective case series study. Medicina oral, patologia oral y cirugia bucal. 2015 Jul;20(4):e480.

39. Shah AK. Short implants - When, where and how? J Int Clin Dent Res Organ 2015; 7:132-7.

40. Huynh-Ba G, Oates TW, Williams MA. Immediate loading vs. early/conventional loading of immediately placed implants in partially edentulous patients from the patients' perspective: A systematic review. Clinical oral implants research. 2018 Oct; 29:255-69.

41. Maluf LR, Tolentino RR, Fernandes Filho PG. IJZ (2018) Immediate Load Implants: Systematic Review on the Clinical Success Assessment and Follow Up. J Interdiscipl Med Dent Sci.;6(232):2.

42. Weerapong K, Sirimongkolwattana S, Sastraruji T, Khongkhunthian P. Comparative study of immediate loading on short dental implants and conventional dental implants in the posterior mandible: A randomized clinical trial. International Journal of Oral \& Maxillofacial Implants. 2019 Jan 1;34(1). 
43. Delal B, Simge T. Biomechanical Evaluation of Short Implants for Prostheses: Review Article. 2020 - 3(2) OAJBS. ID.000239. DOI: $10.38125 /$ OAJBS.000239

44. Bellia E, Audenino G, Ceruti P, Bassi F. Clinical Assessment of Short Implants Retaining Removable Partial Dentures: 4-year Follow-up. International Journal of Oral \& Maxillofacial Implants. 2020 Jan 1;35(1).

45. Hadilou M, Ebrahimi P, Karimzadeh B, Ghaffary A, Gholami L, Fathifar Z. Immediate loading of short implants: A systematic review. Journal of Advanced Periodontology \& Implant Dentistry. 2021 Mar 6;13(1):15-21.

46. Liu W, Cai H, Zhang J, Wang J, Sui L. Effects of immediate and delayed loading protocols on marginal bone loss around implants in unsplinted mandibular implant-retained overdentures: a systematic review and meta-analysis. BMC oral health. 2021 Dec;21(1):1-4.

47. Grant BT, Pancko FX, Kraut RA. Outcomes of placing short dental implants in the posterior mandible: a retrospective study of 124 cases. Journal of oral and maxillofacial surgery. 2009 Apr 1;67(4):713-7.

48. El Mekawy NH, El-Raof El-Negoly SA, El-Awady Grawish M, El-Hawary YM. Intracoronal Mandibular Kennedy Class I Implant-Tooth-Supported Removable Partial Overdenture: A 2-Year Multicenter Prospective Study. International Journal of Oral \& Maxillofacial Implants. 2012 Jun 1;27(3).

49. Wada Y, Yoshimura H, Mikami I. Combination of short implants and a unilateral distal-extension implant-retained partial denture in case of severely reduced ridge height mandibular posterior region: 12-year follow-up. The journal of the academy of clinical dentistry. 2017 May 22;37(1-2):72.

50. Amr Zahran BD, Fouad Al Tayib BD. Clinical and Radiographic Evaluation of Short Dental Implants in Posterior Atrophic Ridges with a Follow-up Period of 1 Year after
Loading: A Controlled Clinical Trial. Custom Emergence Profile Around Implants.:26.

51. Faot F, Marcello-Machado RM, Hermann C, Fontão FN. Splinted wide-short implants in the posterior region of an atrophic mandible opposed by an edentulous maxilla: immediate loading and 1-year follow-up. General dentistry. 2019 Jul 1;67(4):29-33

52. Guida L, Annunziata M, Esposito U, Sirignano M, Torrisi $\mathrm{P}$, Cecchinato D. 6-mm-short and 11-mm-long implants compared in the full-arch rehabilitation of the edentulous mandible: A 3-year multicenter randomized controlled trial. Clinical oral implants research. 2020 Jan; 31(1):64-73.

53. Alysson-Henrique-Neves Ramos GM, Cornacchia EN, Mauricio-Greco Cosso LN, Souza EG. Extra short 4mm implants used to rehabilitation of atrophic posterior mandible. A serial case reports. Journal of Clinical and Experimental Dentistry. 2020 May;12(5):e519.

54. Banihashemrad SA, Gholami M, Ramezani F, Haghdadi F. Evaluation of Stability in Short Implants Compared to Standard Implants using Periotest ${ }^{\circledR}$ : A Pilot Study. Journal of Dental Materials and Techniques. 2020;9(2):63-8.

55. Bellia E, Audenino G, Ceruti P, Bassi F. Clinical Assessment of Short Implants Retaining Removable Partial Dentures: 4-year Follow-up. International Journal of Oral \& Maxillofacial Implants. 2020 Jan 1;35(1).

56. Pardo-Zamora G, Ortiz-Ruíz AJ, Camacho-Alonso F, Martínez-Marco JF, Molina-González JM, Piqué-Clusella N, Vicente-Hernández A. Short Dental Implants $(\leq 8.5 \mathrm{~mm})$ versus Standard Dental Implants ( $\geq 10 \mathrm{~mm}$ ): A One-Year Post-Loading Prospective Observational Study. International Journal of Environmental Research and Public Health. 2021 Jan;18(11):5683.

57. Alkhodary MA, Abdelraheim EA, Elsantawy AH, Al Dahman YH, Al-Mershed M: The development of a composite bone model for training on placement of dental implants. IJHS.2014. in Press. 